Chaire de recherche sur les enjeux économiques intergénérationnels

\title{
Long Term Care
}

Insurance with State-

Dependent Preferences

Philippe De Donder and Marie-Louise Leroux

Cahier de recherche Working paper

Janvier / January 2020

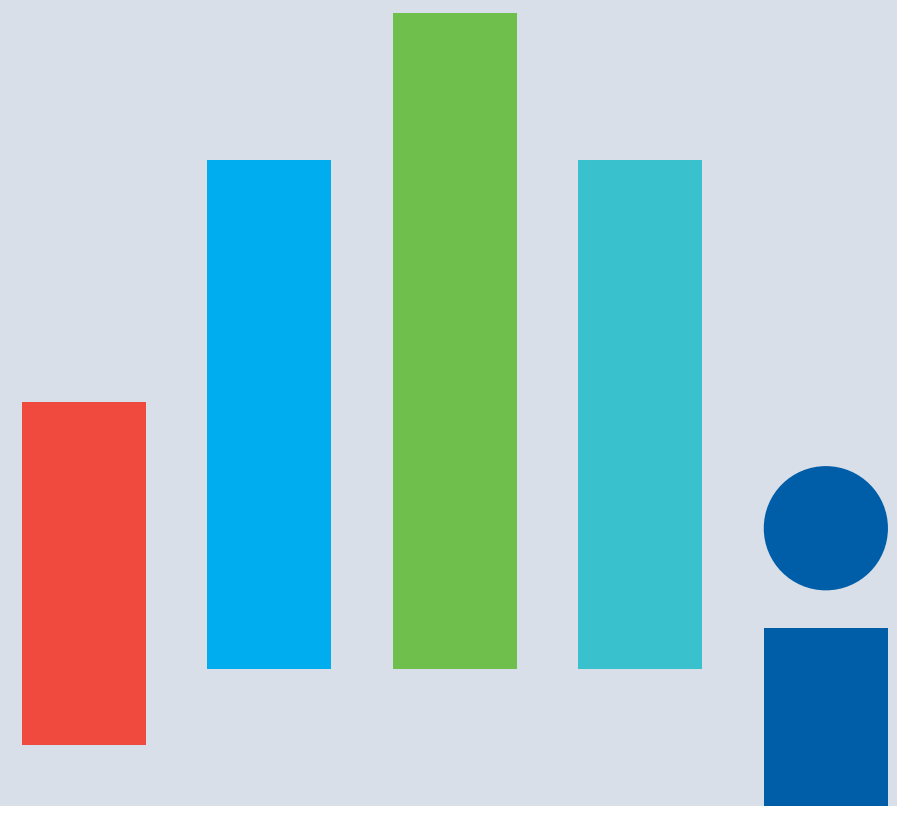




\title{
Chaire de recherche
} sur les enjeux économiques intergénérationnels

\author{
est une chaire multi-institutionnelle qui s'appuie
}

sur un partenariat avec les organisations suivantes :

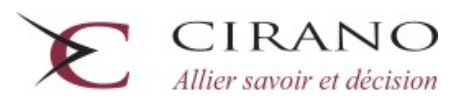

Quetraite

Les opinions et analyses contenues dans les cahiers de recherche de la Chaire ne peuvent en aucun cas être attribuées aux partenaires ni à la Chaire elle-même et elles n'engagent que leurs auteurs.

Opinions and analyses contained in the Chair's working papers cannot be attributed to the Chair or its partners and are the sole responsibility of the authors. 


\title{
Long Term Care Insurance with State-Dependent Preferences ${ }^{1}$
}

\author{
Philippe De Donder ${ }^{2}$ and Marie-Louise Leroux ${ }^{3}$
}

January 7, 2020

${ }^{1}$ The first author acknowledges funding from ANR under grant ANR-17-EURE-0010 (Investissements d'Avenir program) and from the Chair "Marché des risques et création de valeurs, fondation du risque/Scor".

${ }^{2}$ Toulouse School of Economics, CNRS, University of Toulouse Capitole, Toulouse, France. Email: philippe.dedonder@tse-fr.eu

${ }^{3}$ Département des Sciences Economiques, ESG-UQAM. E-mail: leroux.marie-louise@uqam.ca 


\begin{abstract}
We study the demand for actuarially fair Long Term Care (LTC hereafter) insurance in a setting where autonomous agents only care for daily life consumption while dependent agents also care for LTC expenditures. We assume that dependency decreases the marginal utility of daily life consumption. We first obtain that some agents optimally choose not to insure themselves, while no agent wishes to buy complete insurance. We then show that the comparison of marginal utility of income (as opposed to consumption) across health states depends on (i) whether agents do buy LTC insurance at equilibrium or not, (ii) the comparison of the degree of risk aversion for consumption and for LTC expenditures, and (iii) the income level of agents. Our results then offer testable implications that can explain (i) why few people buy Long Term Care insurance and (ii) the discrepancies between various empirical works when measuring the extent of state-dependent preferences for LTC.
\end{abstract}

Keywords: Long Term Care Insurance Puzzle, Actuarially Fair Insurance, Risk Aversion

JEL Codes: D11, I13. 


\section{Introduction}

Population is aging in most developed countries. According to OECD (2011), the fraction of people aged 80 and above is expected to grow from 4\% of the total OECD population in 2010 to $10 \%$ in 2050. This demographic trend creates new challenges for policy makers, as aging implies taking care of an increasing population with very specific health needs, called long term care (hereafter LTC) needs. LTC is defined as "the day-to-day help with activities such as washing and dressing, or help with household activities such as cleaning and cooking" (OECD, 2011). LTC often comes with additional type of support such as medical assistance. Individuals in need of LTC are called dependent.

The costs of LTC are usually large and likely to exhaust most financial resources of the elderly dependent and of his family. For example, Genworth (2018) estimates that the monthly median cost of home care services in the US in 2018 was around US $\$ 4,000$ while that of a semi-private room in a nursing home care was more than US\$ 7,000. The risk of needing LTC is also quite large. Brown and Finkelstein (2009) obtain that between 35\% and 50\% of 65 -year-old Americans will be in need of a nursing home at some point. Hurd et al. (2013) predict a range between $53 \%$ and $59 \%$ of 50 year old individuals who will need LTC services.

Despite an increasing total population that will have a high probability of becoming dependent, most people still do not insure themselves against the risk of LTC. For instance, only $2 \%$ of LTC expenditures are financed by private LTC insurance (LTCI hereafter) in OECD countries, while the figure is $7 \%$ in the US (OECD, 2011). This lack of insurance is referred to as the LTCI puzzle 11 A large body of the economics literature, both empirical and theoretical, has tried to explain that puzzle. Many explanations can be found either on the supply side (adverse selection, rationing effects which increase prices) or on the demand side (substitution with informal family care, risk misperceptions, bequest motives, knowledge of the product and of the LTC costs and institutional support, narrow framing) $!^{2}$

This paper concentrates on another explanation for this lack of insurance. Dependency usually happens at a time in life (at older age, during the retirement period) when individuals enjoy a type of consumption that depends very much on their health status, such as leisure

\footnotetext{
${ }^{1}$ See Pestieau and Ponthiere (2011).

${ }^{2}$ Regarding supply-side explanations, see for example Brown and Finkelstein (2009), Sloan and Norton (1997), Finkelstein and McGarry (2006). Regarding demand-side explanations, see Bonsang (2009), De Donder and Leroux (2014, 2017), Boyer et al. (2019, 2020), Gottlieb and Mitchell (2019) among others.
} 
goods (traveling, attending cultural events, going to restaurants, doing sports, etc.). When dependency strikes, those goods and services get more difficult to be consumed, or may provide less enjoyment. If the marginal utility of those goods decreases with the advent of dependency, individuals may rationally refrain from transferring resources to the dependency state by buying LTCI.

The state-dependency of preferences has long been discussed in the health economics literature, with the seminal contributions of Zeckhauser (1970) and Arrow (1974). To the best of our knowledge, all theory papers on LTCI (see, among others, Cremer and Pestieau, 2014; De Donder and Pestieau, 2017; De Donder and Leroux, 2014; Canta et al., 2016; Klimaviciute and Pestieau, 2018) assume that individuals consume a composite good (including LTC services in case of dependency) and model dependency as the equivalent of a monetary loss. Dependency then results in higher marginal utility than under autonomy and thus to full insurance when available at actuarially fair terms $3^{3}$ Our first contribution is to propose a simple theoretical model disentangling preferences for daily life consumption from preferences for LTC expenditures (including its health services component), with preferences varying with the LTC state, and to study its impact on the demand for LTCI.

The empirical literature on state-dependent preferences in the context of the loss of autonomy has failed so far to generate a consensus. On the one hand, Lillard and Weiss (1997), and Ameriks et al. (2019), find that marginal utility is higher when dependent than when autonomous. On the other hand, Finkelstein et al. (2013), Hong et al. (2013) and Koijen et al. (2016) obtain the opposite result 4 It is important to note that these papers differ in their modeling of income, consumption, and in the scenarii considered. Ameriks et al. (2019) study the marginal utility obtained from a composite consumption good and thus estimate how the marginal utility from income is affected by the advent of dependency, while Hong et al. (2013) separate (nonhealth) consumption from health expenditures. Finkelstein et al. (2013) also distinguish between

\footnotetext{
${ }^{3}$ One exception is Leroux et al. (2019) who assume lower marginal utility of income under dependency together with extra LTC spending so as to ensure that individuals partially insure themselves against dependency. Yet, their paper is quite different from ours since it studies a normative problem in which an ex-post egalitarian social planner wishes to compensate old-age dependent agents as well as short-lived agents for their unluckiness.

${ }^{4}$ Lillard and Weiss (1997) study individuals' saving and consumption decisions at the end of life using a sample of individuals aged 65 and more, so that the bad health status could be interpreted as becoming dependent. Koijen et al. (2016) estimate a health-state dependent utility function to analyze its effect on the observed demand for insurance products. Given their sample selection, their "sick" state may also be interpreted as being in need of LTC. Finkelstein et al. (2013) consider the number of chronic diseases, which are closely linked to the advent of dependency.
} 
consumption of health services and non-health consumption, with only sick individuals deriving utility from health services. The objective of their empirical analysis is to recover an unbiased estimate of how sickness affects the marginal utility of non-health consumption. Their point estimates indicate that a one-standard deviation increase in the number of chronic diseases is associated with a $10 \%-25 \%$ decline in the marginal utility of non health consumption.

Our second contribution is then to reconcile a lower marginal utility of non-health consumption with a larger marginal utility of income, when dependent. As in Hong et al. (2013) and in Finkelstein et al. (2013), we assume that agents derive utility from LTC (including health) services only if they become dependent, in which case the marginal utility of non-LTC consumption is lower. All agents face the same probability of becoming dependent, and differ in income. Before the advent of dependency, they choose how much of an actuarially fair LTCI to buy.

We obtain that agents always buy less than full LTCI, with some agents preferring not to buy any insurance at all. Agents who prefer not to buy any insurance do so because their marginal utility of income is lower when dependent than when autonomous. As for those who buy LTCI, marginal utility of income is larger (resp., smaller) when dependent than when autonomous provided that their most-preferred LTCI amount increases (resp., decreases) with income. We show that the most-preferred LTCI amount may be non-monotone in income.

To shed more light on these results, we then assume that preferences are iso-elastic and we show that low (resp., high) income individuals insure themselves at equilibrium when the elasticity of LTC expenditures to income is lower (resp., higher) than unity. We find generically that no individuals have the same marginal utility of income when dependent and when autonomous. This is in stark contrast with the usual result of full insurance with non state-dependent preferences where being dependent is associated with both smaller absolute utility and higher marginal utility from a single composite consumption good. We predict that one would observe higher marginal utility of income when dependent among the following agents: (i) agents who do not buy LTCI, even though they should (either because they make a mistake, or because such actuarially fair LTCI is not offered, or because they are not aware of its existence), (ii) agents whose most-preferred insurance amount increases with income. The latter group is composed of individuals with different income levels, according to the value of the elasticity of LTC expenditures to income (which itself depends on the comparison between relative risk aversion with respect to consumption and to LTC expenditures): high-income people if this elasticity is larger than 
unity, and some low-income people if this elasticity is smaller than unity. Other agents should exhibit a lower marginal utility of income when dependent than when autonomous.

The paper is organized as follows. Section 2 presents the generic model, including the individual choices, the comparison of marginal utility of income when dependent and when autonomous, and the comparative statics of the individual choices with respect to income. Section 3 introduces iso-elastic utility functions. Section 4 gives some conclusions and policy implications. Most proofs are relegated to an Appendix.

\section{The model}

\subsection{State-dependent utilities}

An individual derives utility both from the consumption $c$ of a "daily-life", non-LTC, good and from her health status $h$. In old age, this agent can be either autonomous, denoted by $a$ or dependent, denoted by $d$. Her health status depends on both whether she is dependent or autonomous, and on the amount of LTC (including health) expenditures $z$ she consumes. The utility of the agent in state $i=\{d, a\}$ is denoted by

$$
U_{i}(c, z)=u_{i}(c)-h_{i}(z)
$$

so that we make the assumption that utility is separable in consumption and in health status, in both states of the world 5

The utility of "daily-life" consumption is state-dependent with

$$
\begin{aligned}
u_{i}^{\prime}(.) & >0, u_{i}^{\prime \prime}(.)<0 \forall i=\{a, d\}, \\
u_{i}^{\prime}(0) & \rightarrow \infty, \\
u_{a}(x) & >u_{d}(x) \text { and } u_{a}^{\prime}(x)>u_{d}^{\prime}(x) .
\end{aligned}
$$

The first line is standard, with increasing and concave utility from consumption, independently of the dependency status, while the third line is the usual Inada condition. The second line states that when autonomous, both the marginal and the absolute utility of consuming an amount $x$ are higher than when dependent. This reflects the observation that "daily life" consumption

\footnotetext{
${ }^{5}$ We assume separable preferences to show how the simplest model with state-dependent preferences can rationalize the low demand for LTCI. Our framework can be generalized to non-separable preferences, but this would complicate the solving of our model without bringing commensurate insights as to the impact of statedependent preferences.
} 
(such as restaurants, travel, clothing, active leisure, etc.) is more enjoyable when in good health than when dependent, as ascertained, among others, by Hong et al. (2013). Beyond LTC, this assumption may also be satisfied for some health troubles (such as the chronic diseases studied by Finkelstein et al. (2013)) but not by those ailments where a treatment either brings the sufferer back to good health, or at least allows her to function normally.

As for LTC (including health) expenditures, we assume for simplicity that an autonomous agent needs no such expenditures, so that $h_{a}(z)=\bar{h}=0, \forall z$ and $h_{a}^{\prime}(z)=0, \forall z$, and autonomous agents always choose $z=0.6$ As for dependent agents, we assume that $h_{d}(z)=h(z) \geq 0$, $h^{\prime}(z) \leq 0, h^{\prime \prime}(z) \geq 0$ and $h^{\prime}(0) \rightarrow-\infty$. In words, LTC expenditures generate infinite utility at the margin when $z=0$, with decreasing marginal utility as $z$ increases (recall that we subtract $h(z)>0$ to obtain the individual's utility).

Therefore, the agent's utility when autonomous is

$$
U_{a}(c, z)=u_{a}(c)
$$

while it corresponds to

$$
U_{d}(c, z)=u_{d}(c)-h(z)
$$

when dependent.

As mentioned in the introduction, most theoretical papers (Cremer and Pestieau, 2014; De Donder and Pestieau, 2017; De Donder and Leroux, 2014; Canta et al., 2016; Klimaviciute and Pestieau, 2018) do not make the distinction between the utility of consuming leisure goods and health expenditures. Instead, these papers model a unique composite good $x$, with $u_{d}(x)=$ $u(x-L), u_{a}(x)=u(x)$ and where $L$ is a monetary-equivalent utility loss from dependency so that $u_{a}(x)>u_{d}(x)$ and $\left.u_{d}^{\prime}(x)>u_{a}^{\prime}(x)\right]^{7}$ To the best of our knowledge, the only exception is Leroux et al. (2019) who assume that the utility in case of dependency is $u_{d}(x-S)$ where $S$ is the (exogenous) amount of health expenditures in case of dependency and where $u_{a}(x)>u_{d}(x)$ and $u_{a}^{\prime}(x)>u_{d}^{\prime}(x)$.

\footnotetext{
${ }^{6}$ The crucial assumption we need is that marginal utility of LTC (including health) expenditures is higher under dependency than under autonomy: $h_{d}^{\prime}(z)>h_{a}^{\prime}(z)$. Assuming that $h_{a}^{\prime}(z)=0$ is then without further loss of generality.

${ }^{7}$ Canta et al. (2016) model the utility under dependence as $u_{d}(x)=(1+\xi) u_{a}(x)-L$ with $\xi>0$, also leading to $u_{d}^{\prime}(x)>u_{a}^{\prime}(x)$.
} 


\subsection{Individual choices}

At the time of taking the decision to get insured against the LTC risk, all agents face the same probability $p \in[0,1]$ of becoming dependent. We assume that there exists a private insurance market against the risk of needing LTC. The insurance market is perfectly competitive (profits of insurance firms are driven to zero) with no loading costs so that agents face an actuarially fair insurance market where they pay a premium $t$ in return for a LTC benefit $R=t / p$ in case of dependency 87 We assume away any kind of market inefficiency to focus on the state-dependency of preferences as the sole rationale for the low demand for LTCI.

We denote an individual by the income $w$ she is endowed with. Agents choose simultaneously the amount of insurance premium $t$ and the amount of LTC expenditures $z$ in case of dependency to maximize their expected utility function $: 9$

$$
E U(t, z)=(1-p) u_{a}\left(c_{a}\right)+p\left[u_{d}\left(c_{d}\right)-h(z)\right]
$$

where $c_{a}=w-t$ is consumption if autonomous while $c_{d}=w-t+\frac{t}{p}-z$ is consumption if dependent.

First-order conditions with respect to LTC expenditures, $z$ and the premium paid, $t$ are ${ }^{10}$

$$
\begin{aligned}
& \frac{\partial E U}{\partial z}=-u_{d}^{\prime}\left(c_{d}\right)-h^{\prime}(z)=0, \\
& \frac{\partial E U}{\partial t}=(1-p)\left[u_{d}^{\prime}\left(c_{d}\right)-u_{a}^{\prime}\left(c_{a}\right)\right] \leq 0 .
\end{aligned}
$$

We denote by $\left(z^{*}, t^{*}\right)$ the solution to this system of two equations, with the corresponding consumption levels $c_{a}^{*}$ and $c_{d}^{*}$. The assumption that $h^{\prime}(0) \rightarrow-\infty$ implies that equation (1) always holds with equality, so that $z^{*}>0$. As we will see shortly, some individuals may decide not to insure at all. We then denote by $z^{0}$ the optimal level of LTC expenditures (satisfying equation 1) when $t=0$. In that case, we denote the consumption bundle as $\left(z^{0}, c_{d}^{0}, c_{a}^{0}\right)$ with $c_{a}^{0}=w>c_{d}^{0}=w-z^{0}$.

Proposition 1 (i) An agent chooses to (not) buy LTCI if $u_{d}^{\prime}\left(w-z^{0}\right)>u_{a}^{\prime}(w)(<)$. (ii) If the agent decides to insure herself, the level of LTCI coverage is incomplete, that is $c_{d}^{*}<c_{a}^{*}$

\footnotetext{
${ }^{8}$ This modeling also corresponds to the case of a non redistributive public LTC insurance.

${ }^{9}$ Assuming a two-period model in which the individual is in good health with certainty and pays a premium in the first period, but may become dependent and obtain a LTC benefit in the second, yields the same results. Also, whether $z$ is chosen at the same time as $t$, or later on when dependency arises, is of no consequence here since we assume away time inconsistency or any other behavioral problem.

${ }^{10}$ Second-order conditions are satisfied.
} 
and $t^{*}<p z^{*}$. (iii) (a) $z^{0} \leq z^{*} \leq z^{0}+t^{*}(1-p) / p$ with strict inequalities iff $t^{*}>0$. (b) $c_{a}^{0} \geq c_{a}^{*}>c_{d}^{*} \geq c_{d}^{0}$ with strict inequalities iff $t^{*}>0$.

Proof. (i) The agent decides to buy insurance if and only if her marginal gain from buying insurance is positive when $t=0$, namely

$$
u_{d}^{\prime}\left(w-z^{0}\right)-u_{a}^{\prime}(w)>0
$$

(ii) We now assume that $u_{d}^{\prime}\left(w-z^{0}\right)>u_{a}^{\prime}(w)$ so that the agent buys LTCI at equilibrium. In that case, the FOC with respect to $t$ holds with equality and $t^{*}$ is defined by

$$
u_{d}^{\prime}\left(w-t^{*}-z^{*}+\frac{t^{*}}{p}\right)=u_{a}^{\prime}\left(w-t^{*}\right)
$$

where $z^{*}$ is defined by $u_{d}^{\prime}\left(w-t^{*}-z^{*}+t^{*} / p\right)=h^{\prime}\left(z^{*}\right)$. Equation (4) together with $u_{d}^{\prime}(x)<u_{a}^{\prime}(x)$ and with the concavity of both $u_{d}$ and $u_{a}$ imply that $c_{d}^{*}<c_{a}^{*}$ and thus that $t^{*}<p z^{*}$.

(iii) See the Appendix.

These results are in stark contrast with the prediction of full insurance for all agents obtained up to now in the theoretical literature on LTCI assuming that marginal utility is higher when dependent than when autonomous. We rather obtain that some agents may not insure at all, and that no agent buys full insurance.

The advent of dependency has two impacts of opposite signs on the demand for insurance. On the one hand, dependency reduces the marginal utility of daily life consumption, so that individuals have no incentive to insure and transfer resources to the bad state of the world. On the other hand, dependent agents bear additional expenses $z^{*}$, inducing them to insure so as to smooth consumption. Depending on which effect dominates, the agent chooses to insure or not. We therefore have a simple explanation as to why agents may not insure themselves against dependency even though they are risk averse and may incur extra expenses when dependent.

Note that the second result in Proposition 1 that $t^{*} / p<z^{*}$ implies that agents optimally buy more LTC expenditures than the transfer received from insurance. This implies that any constraint stated in the insurance contract that insurance payments have to be spent entirely on LTC expenditures is not binding at equilibrium in our model 11

\footnotetext{
${ }^{11}$ For instance, many US LTC insurance plans make transfers corresponding to the reimbursement of LTC expenditures, while Canadian LTC insurance plans make the transfer simply conditional on the advent of at least two limitations in activities of daily living, without strings being attached as to how the transfer has to be used. In our setting, the additional constraints put by US type plans are not binding at equilibrium.
} 
The third result of Proposition 1 shows that part of the insurance transfer, net of the premium paid (i.e., $t^{*} / p-t^{*}$ ), is used to finance increased LTC expenditures while the remainder is used to increase the non-LTC consumption level, so as to partially compensate for the loss in daily-life consumption utility due to dependency.

We are now in position to study the comparison of marginal utilities of income (as opposed to consumption) across dependency states.

\subsection{Marginal utility of consumption versus marginal utility of income}

In case of dependency, the (ex-post) indirect utility of an agent with income $w$ is:

$$
V_{d}(w)=u_{d}\left(w+t^{*}\left(\frac{1}{p}-1\right)-z^{*}\right)-h\left(z^{*}\right)
$$

with $t^{*}$ and $z^{*}$ depending on $w$. In case of autonomy, the indirect utility of the agent is

$$
V_{a}(w)=u_{a}\left(w-t^{*}\right)
$$

Let us first assume that the agent does not insure herself against the LTC risk, so that $t^{*}=0$. In that situation, using the envelope theorem for the choice of LTC expenditures $z_{0}$, the marginal utility of income in case of dependency and of autonomy are equal to

$$
\begin{aligned}
V_{d}^{\prime}(w) & =u_{d}^{\prime}\left(w-z^{0}\right), \\
V_{a}^{\prime}(w) & =u_{a}^{\prime}(w) .
\end{aligned}
$$

Since $t^{*}=0$, we obtain from equation (3) that $V_{d}^{\prime}(w)<V_{a}^{\prime}(w)$.

Suppose now that $t^{*}>0$. Differentiating (5) and (6) with respect to $w$, and using the envelope theorem for $z^{*}$, we obtain

$$
\begin{aligned}
V_{d}^{\prime}(w) & =u_{d}^{\prime}\left(c_{d}^{*}\right)\left[1+\frac{d t^{*}}{d w}\left(\frac{1}{p}-1\right)\right], \\
V_{a}^{\prime}(w) & =u_{a}^{\prime}\left(c_{a}^{*}\right)\left[1-\frac{d t^{*}}{d w}\right] .
\end{aligned}
$$

From the FOC for $t^{*}>0$ (equation 22, we know that $u_{d}^{\prime}\left(c_{d}^{*}\right)=u_{a}^{\prime}\left(c_{a}^{*}\right)$, resulting in the following proposition. 
Proposition 2 If an individual most prefers no insurance $\left(t^{*}=0\right)$, then $V_{d}^{\prime}(w)<V_{a}^{\prime}(w)$. If she rather prefers some insurance $\left(t^{*}>0\right)$, then we have that

$$
\begin{aligned}
& V_{d}^{\prime}(w)>V_{a}^{\prime}(w) \text { if and only if } \frac{d t^{*}}{d w}>0, \\
& V_{d}^{\prime}(w)=V_{a}^{\prime}(w) \text { if and only if } \frac{d t^{*}}{d w}=0, \\
& V_{d}^{\prime}(w)<V_{a}^{\prime}(w) \text { if and only if } \frac{d t^{*}}{d w}<0 .
\end{aligned}
$$

The intuition for this proposition runs as follows. We use the envelope theorem for the choice of LTC expenditures, so that we can concentrate on the impact of higher income on utility via the (non-LTC) consumption channel. If an agent chooses no insurance $\left(t^{*}=0\right)$, this means that the marginal utility of consumption (and hence of income) is lower when dependent than when autonomous. If an agent rather chooses some insurance $\left(t^{*}>0\right)$, then this most-preferred amount of insurance equalizes the marginal utility from consumption across both states. The comparison of the marginal utilities of income then depends on whether more or less resources are transferred from one state to the other as income increases. We then obtain that marginal utility of income is larger (resp., smaller) when dependent than when autonomous if the amount of insurance bought at equilibrium increases (resp., decreases) with income.

Note that there is another circumstance where marginal utility of income is larger when dependent than when autonomous: when individuals would like to buy actuarially fair insurance, but do not because either such insurance does not exist, or because they are not aware of its existence (see Boyer et al. 2019). In that case, equation (3) is satisfied when $t=0$, so that

$$
V_{d}^{\prime}(w)>V_{a}^{\prime}(w)
$$

We then study in the next section the conditions underlying the variation of the amount of insurance bought, $t^{*}$, with income.

\subsection{Comparative statics with respect to $w$}

In this section, we explore how the agent's insurance behavior varies with her income, $w$, as this is crucial to compare the marginal utility of income across states (see Proposition 2). Our results are summarized in the following proposition:

Proposition 3 We obtain that: 


\section{LTC expenditures $z^{*}$ are increasing in $w$.}

2. The amount, $t^{*}$, of LTCI bought may be increasing or decreasing in $w$.

3. Consumption level $c_{d}^{*}, c_{a}^{*}$ increase with $w$.

4. When $t=0$, we have

$$
0<\frac{d c_{d}^{0}}{d w}, \frac{d z^{0}}{d w}<1
$$

Not surprisingly, LTC expenditures are a normal good. Non-LTC consumption is also a normal good, whether the insurance amount is positive (and chosen optimally) or nil. Yet, we find here that the level of LTCI bought may vary non-monotonically with the individual's income. Indeed, as we prove in the Appendix, it crucially depends on the sign of $u_{d}^{\prime \prime}\left(c_{d}^{*}\right)-u_{a}^{\prime \prime}\left(c_{a}^{*}\right)$ and thus, on the signs of third derivatives 12 We then have to introduce functional forms in order to shed more light on who chooses not to insure themselves (Proposition 1) and who has a larger marginal utility of income when dependent (Proposition 2).

Before moving to functional forms, we study for future reference how the most-preferred insurance rate, denoted $\tau^{*}=t^{*} / w$, is affected by income. Proposition 4 shows that the sign of the derivative of $\tau^{*}$ with respect to $w$ depends on the values of the relative risk aversion coefficients with respect to LTC expenditures $R_{r}(z)=-h^{\prime \prime}(z) z / h^{\prime}(z)$ and consumption when autonomous $R_{r}\left(c_{a}\right)=-u_{a}^{\prime \prime}\left(c_{a}\right) c_{a} / u_{a}^{\prime}\left(c_{a}\right)$ as well as on the elasticity of LTC expenditure to income, measured at the preferred choice of the individual, $\varepsilon_{z^{*}, w}$ :

Proposition 4 When strictly positive, the insurance rate $\tau^{*}$ is increasing (resp. decreasing) in w when $R_{r}\left(z^{*}\right) \varepsilon_{z^{*}, w}-R_{r}\left(c_{a}^{*}\right)<0$ (resp. $>$ ).

Proof. See the Appendix.

The role of risk aversion runs as follows. A large risk aversion on the consumption dimension when autonomous means that the function $u_{a}(c)$ is very concave, so that an increase in income will induce the agent to transfer a larger fraction of his income to the dependency state. Analogously, a large risk aversion on the LTC expenditure dimension means that the function $h(z)$ is very convex, so that the agent does not wish to increase his LTC expenditures by much and decreases the share of his income devoted to LTCI.

\footnotetext{
${ }^{12}$ Note that assuming prudence (i.e., that $u^{\prime \prime \prime}()>$.0 ) is not sufficient to sign the derivative of $t^{*}$ with respect to $w$. In the special case where $u_{d}^{\prime \prime \prime}(x)=u_{a}^{\prime \prime \prime}(x)=0, t^{*}$ unambiguously increases with $w$.
} 
As for the role of the elasticity of LTC expenditures to income, note that, for given values of $R_{r}(z)$ and $R_{r}\left(c_{a}\right)$, a low value of $\varepsilon_{z, w}$ means that the individual has to transfer more income to the dependency state if she wants to increase her LTC expenditures-i.e., she has to increase the share $\tau^{*}$ of LTCI in her income when autonomous.

Finally, note that $\tau^{*}$ increasing with $w$ implies that $t^{*}$ increases with $w$, while $\tau^{*}$ decreasing with $w$ is compatible with $t^{*}$ either increasing or decreasing with $w$.

\section{Iso-elastic utility functions}

The introduction of the widely used iso-elastic functional form (as in Becker et al., 2005; Finkelstein et al., 2013; Hong et al., 2015; Ameriks et al. (2019)) will allow us to shed more light on Propositions 1 and 2 .

\subsection{State-dependent preferences for non-LTC consumption}

We assume the following form for state-dependent preferences for non-LTC consumption.

\section{Assumption 1}

$$
u_{d}(x)=\gamma u_{a}(x)
$$

where $\gamma \in] 0,1[$ is the same for all agents, with

$$
u_{a}(x)=\frac{x^{1-\varepsilon}}{1-\varepsilon}
$$

We now show that how the elasticity of LTC expenditures to income compares with unity determines both whether low or high income individuals choose not to buy LTCI, and whether LTC expenditures as a share of income increase or decrease with income when agents do not buy LTCI (either because their most-preferred level is nil, or because such insurance is not available).

Proposition 5 When Assumption 1 holds, $\varepsilon_{z^{0}, w}=\left(d z^{0} / d w\right)\left(w / z^{0}\right)<1$ (resp., >1) implies that (i) agents with an income lower than a threshold $\tilde{w}$ (resp., higher) defined in the Appendix insure themselves at equilibrium, and that (ii) $z^{0} / w$ decreases (resp., increases) with $w$.

Proof. See the Appendix.

The intuition for this result runs as follows. Recall that agents buy insurance if their marginal utility of (non-LTC) consumption when dependent is larger than when autonomous, in the case 
where LTC expenditures are financed from their own resources (i.e., at level $z^{0}$ ), see equation (3). Recall also that the advent of dependency has two impacts of opposite signs on the demand for LTCI, as (i) it reduces the marginal utility of non-LTC consumption (depressing the demand for LTCI) while (ii) increasing the need for LTC expenditures, and thus decreasing the income available for the non-LTC consumption good (increasing the demand for LTCI). If the elasticity of LTC expenditures to income, $\varepsilon_{z^{0}, w}$, is smaller than one, then higher income agents don't increase much their demand for LTC expenditures, $z^{0}$, muting the second effect above and resulting in high income agents preferring not to buy LTCI (part (i) of the above proposition). At the same time, the share of LTC expenditures in income $w$ decreases (part (ii) of the above proposition). The opposite occurs when $\varepsilon_{z^{0}, w}>1$.

Observe that Proposition 5 does not depend on any functional form assumption for the utility obtained from LTC expenditures when dependent.

\subsection{Preferences for LTC expenditures}

We now introduce a functional form for the benefit obtained from LTC expenditures when dependent.

\section{Assumption 2}

$$
h(z)=\delta \frac{x^{1-\alpha}}{1-\alpha} .
$$

The following lemma will prove useful to find the conditions underlying the variation of $t^{*}$ with respect to $w$.

Lemma 1 When Assumptions 1 and 2 hold, the elasticity of LTC expenditures with respect to income is such that, for $z=\left\{z^{0}, z^{*}\right\}$, (i) If $\varepsilon=\alpha, \varepsilon_{z, w}=1$, (ii) If $\varepsilon>\alpha, \varepsilon_{z, w}>1$, (iii) If $\varepsilon<\alpha, \varepsilon_{z, w}<1$.

Under Assumptions 1 and 2, we obtain that $R_{r}(z)=\alpha, \forall z$, and $R_{r}(c)=\varepsilon, \forall c$. If $\alpha>\varepsilon$, agents are more averse to variations in LTC expenditures $z$ than in consumption $c$. The choice of $z$ is then less sensitive to variations in income $w$ than the choice of consumption $c$, resulting in a low elasticity of LTC expenditures to income.13

The next proposition studies how $\tau^{*}=t^{*} / w$ is affected by $w$ with iso-elastic utilities.

\footnotetext{
${ }^{13}$ To see this, we fully differentiate eq. [1] with respect to $w$,

$$
-u_{d}^{\prime \prime}\left(c_{d}^{*}\right) \frac{d c_{d}^{*}}{d w}-h^{\prime \prime}\left(z^{*}\right) \frac{d z^{*}}{d w}=0,
$$
}


Proposition 6 When Assumptions 1 and 2 hold, we obtain:

(i) If $\alpha=\varepsilon$, (a) $t^{*}=0$ if $\delta^{1 / \varepsilon}+\gamma^{1 / \varepsilon} \leq 1$ and (b) $t^{*}$ is linearly increasing in $w$, if $\delta^{1 / \varepsilon}+\gamma^{1 / \varepsilon}>1$.

(ii) If $\alpha<\varepsilon, t^{*}=0$ if $w<\tilde{w}$ (defined in the proof of Proposition 5), and $t^{*} / w$ is increasing for $w>\tilde{w} \geq 0$.

(iii) If $\alpha>\varepsilon$ then $t^{*} / w$ decreases with $w$ up to the threshold $\tilde{w}$ above which $t^{*}=0$.

Let us first study the reasons why agents decide to insure or not, depending on $\alpha \gtrless \varepsilon$. The first part of Proposition 6 shows that, when the relative risk aversion is the same for consumption and for LTC expenditures $\left(R_{r}\left(z^{*}\right)=\alpha=\varepsilon=R_{r}\left(c_{a}^{*}\right)\right)$, all agents want to spend the same proportion of their income on LTCI 14 Whether this share is positive depends on whether the marginal utilities of consumption and of LTC expenditures when dependent are large enough (namely, whether $\delta^{1 / \varepsilon}+\gamma^{1 / \varepsilon}$ is larger than one or not).

As for the second part of Proposition 6, Lemma 1 has shown that $\varepsilon_{z^{0}, w}>1$ if $\varepsilon>\alpha$, while Proposition 5 has shown that in this case rich people (i.e., with income larger than $\tilde{w}$ ) insure themselves. Symmetrically for the third part of Proposition 6, Lemma 1 has shown that $\varepsilon_{z^{0}, w}<1$ if $\varepsilon<\alpha$, while Proposition 5 has shown that in this case poor people (i.e., with income smaller than $\tilde{w}$ ) insure themselves.

We now move to the comparative statics of $\tau^{*}$ with respect to $w$ when $\tau^{*}>0$. Note first that Proposition 4 is of little help, since Lemma 1 implies that $\varepsilon_{z^{*}, w}>1$ when $\alpha=R_{r}(z)<\varepsilon=R_{r}(c)$ under Assumptions 1 and 2, Lemma 1 shows that $\alpha=R_{r}(z)>\varepsilon=R_{r}(c)$ implies that $\varepsilon_{z^{*}, w}<1$. Because of the large curvature of the utility for LTC expenditures, a higher income $w$ translates into a small increase in $z$. This small increase in $z$ can then be financed by a smaller share of LTCI premium in income, so that $\tau^{*}$ decreases with $w$. Observe that this complements Proposition 5 which has shown that low income agents insure themselves at equilibrium when $\varepsilon_{z^{0}, w}<1$ (which by Lemma 1 corresponds to $\alpha>\varepsilon$ ). The opposite occurs when $\alpha<\varepsilon$ in which and we obtain after some rearrangements that

$$
\varepsilon_{c_{d}^{*}, w}=\frac{R_{r}\left(z^{*}\right)}{R_{r}\left(c_{d}^{*}\right)} \varepsilon_{z^{*}, w}
$$

Since $R_{r}\left(c_{d}^{*}\right)=\varepsilon$ and $R_{r}\left(z^{*}\right)=\alpha$ under Assumptions 1 and 2 we have that $\varepsilon_{c_{d}^{*}, w}>\varepsilon_{z^{*}, w}$ if $\alpha>\varepsilon$.

${ }^{14}$ Note that this is a special case of Proposition 4 since Lemma 1 has shown that $\varepsilon_{z^{*}, w}=1$ if $\varepsilon=\alpha$ under Assumptions 1 and 2 
a large increase in $z$ for richer agents has to be financed by an increase in the share of their income devoted to LTCI.

Our final proposition shows that the comparison of marginal utilities of income across states depends on (i) whether LTCI is bought at equilibrium, (ii) the comparison of risk aversion levels for consumption and for LTC expenditures, and (iii) the income level of agents.

Proposition 7 When Assumptions 1 and 2 hold, we have that

(i) $V_{d}^{\prime}(w)>V_{a}^{\prime}(w)$ in the following cases:

(a) $t=0$ but $t^{*}>0$,

(b) $\alpha<\varepsilon$ and $w>\tilde{w}$ (so that $t^{*}>0, d \tau^{*} / d w \geq 0$ and thus $d t^{*} / d w \geq 0$ ),

(c) $\alpha>\varepsilon, w<\tilde{w}$, and $d t^{*} / d w>0$,

(d) $\alpha=\varepsilon$ and $\delta^{1 / \varepsilon}+\gamma^{1 / \varepsilon}>1$.

(ii) $V_{d}^{\prime}(w)<V_{a}^{\prime}(w)$ in the following cases:

(a) $t^{*}=0$,

(b) $\alpha>\varepsilon, w<\tilde{w}$, and $d t^{*} / d w<0$.

(iii) $V_{d}^{\prime}(w)=V_{a}^{\prime}(w)$ never happens generically.

Proof. See the Appendix.

Marginal utility from income is larger when dependent than when autonomous for two sets of agents. First, those who buy no LTCI while they should. Second, those whose (positive) mostpreferred LTCI premium increases with income (see Proposition 2). With iso-elastic preferences, we know who these individuals are: rich people if $\alpha<\varepsilon$, some poor people if $\alpha>\varepsilon$, and all individuals if $\alpha=\varepsilon$ while $\delta^{1 / \varepsilon}+\gamma^{1 / \varepsilon}>1$. The case where the marginal utility of income is the same when dependent and when autonomous never happens generically (more precisely, it may only happen when $\alpha>\varepsilon$ and when $t^{*}$ is non monotone in $w$, precisely for the agents whose income level is such that $\left.d t^{*} / d w=0\right) \sqrt[15]{ }$ For the other agents, marginal utility of income is smaller when dependent than when autonomous.

\footnotetext{
${ }^{15}$ We have performed a large number of simulations with CES preferences, and the only cases where $t^{*}>0$ is non monotone (increasing or decreasing) with $w$ are such that $t^{*}$ is first increasing then decreasing in $w$. We then obtain that $d t^{*} / d w=0$ for at most one single level of $w$.
} 


\section{Conclusions}

We have developed a model where individuals choose how much actuarially fair LTCI they wish to buy, in a setting where autonomous agents only care about daily-life consumption while dependent agents care about both consumption and LTC expenditures. We assume from the outset that the marginal utility of non-LTC consumption is lower when dependent than when autonomous, as obtained by Finkelstein et al. (2013) and Brown et al. (2016), and in stark contrast with most of the literature on LTC. We then study the consequences of this assumption for the demand for LTCI insurance, and for the comparison of marginal utilities of income (as opposed to non-LTC consumption) across health states.

We first obtain that some individuals optimally choose not to buy any LTCI, while no one buys full insurance. We then show that the comparison of marginal utility of income across health states depends on (i) whether agents do buy LTCI at equilibrium, (ii) the comparison of the degree of risk aversion for consumption and for LTC expenditures, and (iii) the income level of agents. More precisely, our model predicts higher marginal utility of income when dependent among the following agents: (i) agents who do not buy LTCI, even though they should (either because they make a mistake, or because such actuarially fair LTCI is not offered, or because they are not aware of its existence), (ii) agents whose most-preferred LTCI premium increases with income. The latter group is composed of individuals whose income is above a threshold income (which may be zero) if their relative risk aversion is larger for consumption than for LTC expenditures, and of some individuals whose income is below a threshold in the opposite case. Other agents should exhibit a lower marginal utility of income when dependent than when autonomous. Finally, although the ratio of most-preferred LTCI premium to income is monotone in income with iso-elastic utilities, this may not be the case for the LTCI premium itself.

The model we propose, while very simple, then delivers testable implications which differ starkly from the ones obtained under the classical assumption of higher marginal utility from consumption when a damage occurs, and may then shed light on both the LTCI puzzle and the seemingly contradictory results obtained by the empirical literature on health dependent utilities in LTC. 


\section{References}

[1] Ameriks J., J. S. Briggs, A. Caplin, M. D. Shapiro and C. Tonetti, 2019, Long-term-care insurance puzzle: Modeling and measurement, Journal of Political Economy, forthcoming.

[2] Arrow, Kenneth, 1974, Optimal Insurance and Generalized Deductibles, Scandinavian Actuarial Journal, 1974, 1-42. Reprinted in Collected Papers of Kenneth J. Arrow, Vol. 3. Harvard University Press, 1984, pp. 212-260.

[3] Becker, G., Philipson, T., Soares, R., 2005, The quantity and quality of life and the evolution of world inequality, American Economic Review, 95 (1): 277-291.

[4] Bonsang, E., 2009, Does informal care from children to their elderly parents substitute for formal care in Europe? Journal of Health Economics, 28(1), 143-154.

[5] Boyer, M.M., P. De Donder, C. Fluet, M-L. Leroux, P-C. Michaud, 2019, Long Term Care Risk misperceptions, Geneva Papers on Risk and Insurance; Issues and Practices, 44(2), $183-215$.

[6] Boyer, M.M., P. De Donder, C. Fluet, M-L. Leroux, P-C. Michaud, 2020, Long-term Care Insurance : Information Frictions and Selection, American Economic Journal: Economic Policy, forthcoming.

[7] Brown, J. R. and Finkelstein, A., 2009, The Private Market for Long-Term Care Insurance in the United States: A Review of the Evidence. Journal of Risk and Insurance, 76(1), 5-29.

[8] Brown, Jeffrey R., Gopi Shah Goda, and Kathleen McGarry, 2016, Heterogeneity in StateDependent Utility: Evidence from Strategic Surveys, Economic Inquiry 54.2 : 847-861.

[9] Canta C., P. Pestieau, and E. Thibault, 2016, Long-term care and capital accumulation: the impact of the State, the market and the family, Economic Theory, 61, 755-785.

[10] Cremer H. and P. Pestieau, 2014, Social long-term care insurance and redistribution, International Tax and Public Finance, 21, 955-974.

[11] De Donder P., and M-L. Leroux, 2014, Behavioral Biases and Long-Term Care Insurance: A Political Economy Approach, B.E Journal of Economic Analysis and Policy, 14(2), 551-575. 
[12] De Donder P., and M-L. Leroux, 2017, The political choice of social long term care transfers when family gives time and money, Social Choice and Welfare, 49 (3-4), 755-786.

[13] De Donder P., and P. Pestieau, 2017, Private, social, and self-insurance for long-term care in the presence of family help, Journal of Public Economic Theory, 19(1), 18-37.

[14] Finkelstein, A. and McGarry, K., 2006, Multiple Dimensions of Private Information: Evidence from the Long-Term Care Insurance Market, American Economic Review, 96(4), 938-958.

[15] Finkelstein A., E. Luttmer, and M. Notowidigdo, 2013, What good is wealth without health? The effect of health on the marginal utility of consumption, Journal of the European Economic Association, 11, 221-258.

[16] Genworth, 2018, Cost of Care Survey 2018. Available at https://www.genworth.com/ aging-and-you/finances/cost-of-care.html

[17] Gottlieb D. and O.Mitchell, 2019, Narrow framing and long term care insurance, The Journal of Risk and Insurance, https://doi.org/10.1111/jori.12290

[18] Hong, J. H., J. Pijoan-Mas, and J.-V. Rios-Rull, 2013, Health Heterogeneity and Preferences, working paper.

[19] Hurd, M. D., Michaud, P.-C., and Rohwedder, S., 2013, The Lifetime Risk of Nursing Home Use. In Discoveries in the Economics of Aging, pages 81-109. University of Chicago Press.

[20] Klimaviciute, J. and P. Pestieau, 2018, Long-term care social insurance: How to avoid big losses?, International Tax and Public Finance, 25(1), 99-139.

[21] Koijen, R. S. J., S. Van Nieuwerburgh, and M. Yogo, 2016, Health and Mortality Delta: Assessing the Welfare Cost of Household Insurance Choice, Journal of Finance, LXXI, 2, 957-1010.

[22] Leroux M-L., G. Ponthière and P. Pestieau, 2019, Fair Long-Term Care Insurance, CESifo Working Paper \#7660.

[23] Lillard, L. A. and Y. Weiss, 1997, Uncertain Health and Survival: Effects on End-of-Life Consumption, Journal of Business \& Economic Statistics, 15(2), 254-268. 
[24] OECD (2011). Help Wanted? Providing and Paying for Long-Term Care, available at http://www.oecd.org/document/23/0,3746, en_2649_37407_47659479_1_1_1_ $37407,00 . \mathrm{html}$

[25] Pestieau, P., Ponthiere, G., 2011, The Long Term Care Insurance Puzzle, in J. Costa-Font and C. Courbage (eds.): Financing Long Term Care in Europe: Institutions, Markets and Models, Palgrave Macmillan, London, 41-52.

[26] Sloan, F. A. and Norton, E. C., 1997, Adverse Selection, Bequests, Crowding Out, and Private Demand for Insurance: Evidence from the Long-term Care Insurance Market, Journal of Risk and Uncertainty, 15(3), 201-219.

[27] Zeckhauser, Richard (1970). "Medical Insurance: A Case Study of the Tradeoff Between Risk Spreading and Appropriate Incentives." Journal of Economic Theory, 2, 10-26.

\section{Appendix}

\section{Proof of Proposition 1}

(iii) (a) If $t^{*}=0$, we have by definition that $z^{0}=z^{*}=z^{0}+t^{*}(1-p) / p$. Assume then that $t^{*}>0$. Assume by contradiction that $z^{0}>z^{*}$. Comparing the equation (1) with $z^{*}$ and $z^{0}$, we obtain that

$$
\begin{aligned}
-h^{\prime}\left(z^{0}\right) & <-h^{\prime}\left(z^{*}\right) \Rightarrow u_{d}^{\prime}\left(w-z^{0}\right)<u_{d}^{\prime}\left(w-z^{*}+t^{*}(1-p) / p\right) \\
& \Leftrightarrow w-z^{0}>w-z^{*}+t^{*}(1-p) / p \\
& \Leftrightarrow z^{*}>z^{0}+t^{*}(1-p) / p
\end{aligned}
$$

which contradicts that $z^{0}>z^{*}$. Proceeding similarly then shows that $z^{0}<z^{*}$ implies that $z^{*}<z^{0}+t^{*}(1-p) / p$.

(b) $t^{*}=0$, we have by definition that $c_{a}^{0}=c_{a}^{*}=w>c_{d}^{*}=w-z^{*}=c_{d}^{0}=w-z^{0}$. Assume then that $t^{*}>0$. We have that $c_{a}^{0}=w>c_{a}^{*}=w-t^{*}>c_{d}^{*}=w+t^{*}(1-p) / p-z^{*}=w-t^{*}+\left(t^{*} / p-z^{*}\right)$ since Proposition 1 (ii) has shown that $z^{*}>t^{*} / p$. Finally, $c_{d}^{*}>c_{d}^{0}$ since $z^{*}<z^{0}+t^{*}(1-p) / p$.

\section{Proof of Proposition 3}

In order to find the variation of health expenditure with income, we apply Cramer's rule on equations (1) and (2). We obtain that $0<d z^{*} / d w<1 / p$. 
In order to find the sign of $d t^{*} / d w$, we fully differentiate equations (1) and (2) with respect to $w$. Substituting for $d z / d w$, after tedious computations, we find that:

$$
\frac{d t^{*}}{d w}=\frac{h^{\prime \prime}\left(z^{*}\right)\left[u_{d}^{\prime \prime}\left(c_{d}^{*}\right)-u_{a}^{\prime \prime}\left(c_{a}^{*}\right)\right]+u_{a}^{\prime \prime}\left(c_{a}^{*}\right) u_{d}^{\prime \prime}\left(c_{d}^{*}\right)}{h^{\prime \prime}\left(z^{*}\right) u_{d}^{\prime \prime}\left(c_{d}^{*}\right)\left(1-\frac{1}{p}\right)+u_{a}^{\prime \prime}\left(c_{a}^{*}\right)\left(u_{d}^{\prime \prime}\left(c_{d}^{*}\right)-h^{\prime \prime}\left(z^{*}\right)\right)}<1
$$

The expression in the denominator is positive. The sign on the numerator is unclear. The last term is positive, $h^{\prime \prime}\left(z^{*}\right)>0$ while $u_{d}^{\prime \prime}\left(c_{d}^{*}\right)-u_{a}^{\prime \prime}\left(c_{a}^{*}\right) \gtrless 0$ depending on the specific forms of $u_{a}($. and $u_{d}($.$) , as well as on the specific levels of c_{a}^{*}$ and $c_{d}^{*}$. If $u_{d}^{\prime \prime}\left(c_{d}^{*}\right)-u_{a}^{\prime \prime}\left(c_{a}^{*}\right)>0, d t^{*} / d w>0$. Otherwise, $d t^{*} / d w \gtrless 0$.

3. Since $z^{*}$ increases with $w$, the FOC for $z$ (equation (1)) implies that $c_{d}^{*}$ increases with $w$ as well. Using the FOC for $t$ (equation (2)), we obtain that $c_{a}^{*}=w-t^{*}$ increases with $w$, so that $d t^{*} / d w<1$. This in turn implies that

$$
\frac{d c_{d}^{*}}{d w}=1+\frac{d t^{*}}{d w} \frac{1-p}{p}-\frac{d z^{*}}{d w}<1+\frac{1-p}{p}-\frac{d z^{*}}{d w} .
$$

Since $d c_{d}^{*} / d w>0$, we obtain that $d z^{*} / d w<1 / p$.

4. Applying the implicit function theorem to the FOC for $z$ (equation (1D) when $t=0$, we obtain

$$
\frac{d z^{0}}{d w}=\frac{u_{d}^{\prime \prime}\left(c_{d}^{0}\right)}{u_{d}^{\prime \prime}\left(c_{d}^{0}\right)-h^{\prime \prime}\left(z^{0}\right)} \in[0,1] .
$$

This in turn implies that $d c_{d}^{0} / d w \in[0,1]$.

\section{Proof of Proposition 4}

Changing notations for $\tau=t / w$ and replacing (1) into (2), we obtain:

$$
-h^{\prime}\left(z^{*}\right)-u_{a}^{\prime}\left(w\left(1-\tau^{*}\right)\right)=0
$$

Fully differentiating this expression with respect to $w$, we get

$$
\frac{d \tau^{*}}{d w}=\frac{1}{w u_{a}^{\prime \prime}\left(c_{a}^{*}\right)}\left[h^{\prime \prime}\left(z^{*}\right) \frac{d z^{*}}{d w}+u_{a}^{\prime \prime}\left(c_{a}^{*}\right)\left(1-\tau^{*}\right)\right] .
$$

From proposition 3, we have that $d z^{*} / d w>0$. Using (7) and rearranging terms, we obtain that

$$
\frac{d \tau^{*}}{d w}=\frac{u_{a}^{\prime}\left(c_{a}^{*}\right)}{w^{2} u_{a}^{\prime \prime}\left(c_{a}^{*}\right)}\left[R_{r}\left(z^{*}\right) \varepsilon_{z^{*}, w}-R_{r}\left(c_{a}^{*}\right)\right]
$$

where $R_{r}(c)=-u^{\prime \prime}(c) c / u^{\prime}(c)$ and $R_{r}(z)=-h^{\prime \prime}(z) z / h^{\prime}(z)$. 


\section{Proof of Proposition 5}

(i) Using equation (3), we obtain that $t^{*}>0$ if and only if

$$
\gamma>\left(\frac{w-z^{0}}{w}\right)^{\varepsilon}
$$

Denote by $\tilde{w}$ the value of $w$ equalizing both sides of (8), namely

$$
\gamma\left(\tilde{w}-z^{0}\right)^{-\varepsilon}-\tilde{w}^{-\varepsilon}=0
$$

Observe that the right hand side of (8) is increasing (resp., decreasing) in $w$ if and only if the elasticity of health expenditure to income, $\varepsilon_{z^{0}, w}=\left(d z^{0} / d w\right)\left(w / z^{0}\right)$, is smaller (resp., larger) than one, so that the value of $\tilde{w}$ is unique when it exists. When $\varepsilon_{z_{0}, w}<1$, agents with $w<\tilde{w}$ insure themselves, while agents with $w>\tilde{w}$ insure themselves if $\varepsilon_{z_{0}, w}>1$. Note that we set $\tilde{w}=+\infty$ if (i) (8) is satisfied for all agents (i.e., including the highest income) when $\varepsilon_{z_{0}, w}<1$ (so that everyone insures at equilibrium) or if (8) is not satisfied, even for the highest income when $\varepsilon_{z_{0}, w}>1$ (so that no one insures at equilibrium). Alternatively, we set $\tilde{w}=0$ if (i) (8) is satisfied for no agent (i.e., including the lowest income) when $\varepsilon_{z_{0}, w}<1$ (so that no one insures at equilibrium) or if (8) is satisfied for all agents (including the lowest income) when $\varepsilon_{z_{0}, w}>1$ (so that everyone insures at equilibrium).

(ii)

$$
\frac{d z^{0} / w}{d w}=\frac{z^{0^{\prime}} w-z^{0}}{w^{2}}
$$

so that

$$
\begin{aligned}
\frac{d z^{0} / w}{d w} & <0 \Leftrightarrow z^{0^{\prime}} w<z^{0} \\
& \Leftrightarrow \varepsilon_{z^{0}, w}<1 .
\end{aligned}
$$

\section{Proof of Lemma 1}

Assume first that $t^{*}>0$. Let us make a change of variables in (1) and (2) with $\tau=t^{*} / w$ and $\bar{z}=z^{*} / w:$

$$
\begin{aligned}
-\gamma\left(1-\tau+\frac{\tau}{p}-\bar{z}\right)^{-\varepsilon}+\delta \bar{z}^{-\alpha} w^{\varepsilon-\alpha} & =0 \\
\gamma\left(1-\tau+\frac{\tau}{p}-\bar{z}\right)^{-\varepsilon}-(1-\tau)^{-\varepsilon} & =0
\end{aligned}
$$


when $t^{*}$ is interior. Applying Cramer's rule on the above two equations, $d \bar{z} / d w<0$ if $\varepsilon>\alpha$. The reverse is true for $\varepsilon<\alpha$. Recognizing that $d \bar{z} / d w$ can be rewritten as

$$
\frac{d \bar{z}}{d w}=\frac{d\left(z^{*} / w\right)}{d w}=\frac{z^{*}}{w^{2}}\left[\varepsilon_{z^{*}, w}-1\right]
$$

it is straightforward to show that $d \bar{z} / d w>0,<0,=0$ when $\alpha>,<,=\varepsilon$ implies that $\varepsilon_{z^{*}, w}>,<$ ,$=1$.

Assume now that $t^{*}=0$, the FOC on $z$ can be rewritten as follows

$$
-\gamma\left[w-z^{0}\right]^{-\varepsilon}+\delta\left(z^{0}\right)^{-\alpha}=0
$$

Fully differentiating this expression with respect to $w$ yields the following equality:

$$
\frac{\varepsilon}{c_{d}}\left[1-\frac{d z^{0}}{d w}\right]^{-}-\frac{\alpha}{z^{0}} \frac{d z^{0}}{d w}=0
$$

Rearranging terms, we obtain that

$$
\varepsilon_{z^{0}, w}=\frac{d z^{0}}{d w} \frac{w}{z^{0}}=\frac{\varepsilon w}{\varepsilon z+\alpha c_{d}}=\frac{c_{d}+z}{z+\frac{\alpha}{\varepsilon} c_{d}} .
$$

which yields Lemma 1 for $\varepsilon_{z^{0}, w}$.

\section{Proof of Proposition 6}

(i) (a): When $t^{*}=0$, equation (2) takes the following form:

$$
\gamma\left(w-z^{0}\right)^{-\varepsilon}-w^{-\varepsilon} \leq 0
$$

From eq. (1), we also obtain that

$$
z^{0}=\frac{\delta^{1 / \varepsilon}}{\delta^{1 / \varepsilon}+\gamma^{1 / \varepsilon}} w
$$

which we insert into inequality 11 to obtain that it is satisfied if $\delta^{1 / \varepsilon}+\gamma^{1 / \varepsilon} \leq 1$.

(i) (b): Using the functional forms in FOCs (1) and (2) and setting $\alpha=\varepsilon$, we obtain that $c_{d}^{*} / c_{a}^{*}=\gamma^{1 / \varepsilon}, z^{*} / c_{d}^{*}=(\delta / \gamma)^{1 / \varepsilon}$ and $z^{*} / c_{a}^{*}=\delta^{1 / \varepsilon}$. We then apply Cramer's rule on the FOCs and find that $d t^{*} / d w>0$ is independent of $w$.

(ii) and (iii): The threshold productivity $\tilde{w}$ is defined in the proof of Proposition 5 .

Applying Cramer's rule on (9) and (10), one finds that $d \tau^{*} / d w>0$ if $\varepsilon>\alpha$ and $d \tau^{*} / d w<0$ if $\varepsilon<\alpha$. 


\section{Proof of Proposition 7}

(i) (a) follows from the commentary after Proposition 2 regarding agents who would like to buy insurance and do not do so because they are not aware of its existence.

(i) (b) follows from Propositions 2 and 6 (ii)

(i) (c) follows from Propositions 2 and 6 (iii)

(i) (d) follows from Propositions 2 and 6 (i)(b)

(ii) (a) follows from Proposition 2

(ii) (b) follows from Propositions 2 and 6 (iii)

(iii) The only case where $V_{d}^{\prime}(w)=V_{a}^{\prime}(w)$ is when $t^{*}>0$ and $d t^{*} / d w=0$, which can only happen for some values of $w$ when $\alpha>\varepsilon$ so that $d \tau^{*} / d w$ is decreasing in $w$. All other cases are covered either in part (i) or (ii) of the Proposition. 